\title{
Using X-ray Spectromicroscopy for Operando Characterization of Li-S Batteries
}

\author{
Elizabeth C. Miller $^{1, *}$, Sophia J. Kurdziel ${ }^{1}$, Khloe N. Heath ${ }^{1}$, Robert M. Kasse ${ }^{1,2}$, Brian R. Perdue ${ }^{3}$ and \\ Michael F. Toney ${ }^{1}$ *
}

1. Stanford Synchrotron Radiation Lightsource, SLAC National Accelerator Laboratory, Menlo Park, CA, USA

2. Department of Materials Science \& Engineering, Stanford University, Stanford, CA, USA

3. Joint Center for Energy Storage Research, Sandia National Laboratories, Albuquerque, NM, USA

* Corresponding authors, ecmiller@slac.stanford.edu; mftoney@slac.stanford.edu

Li-S batteries are a "beyond Li-ion" technology that delivers large capacity (1672 $\left.\mathrm{mAh} \mathrm{g}^{-1}\right)$ while also being earth-abundant, lightweight, and low cost. Li-S achieves high capacity via a chemical transformation mechanism rather than $\mathrm{Li}$ intercalation as in Li-ion. Elemental sulfur $\mathrm{S}_{8}$ is reduced through a series of soluble chain-like polysulfides ( $\mathrm{LiPS} ; \mathrm{Li}_{2} \mathrm{~S}_{\mathrm{x}}, 2 \leq \mathrm{x} \leq 8$ ) to a final solid discharge product $\mathrm{Li}_{2} \mathrm{~S}$; this reaction is reversed on charging. However, Li-S suffers from unrealized theoretical capacity and capacity fade due to loss mechanisms that are not well understood. LiPS, while necessary for battery operation, are likely a critical factor in degradation processes; thus, deciphering the speciation and spatial distribution of dissolved LiPS in the electrodes and electrolyte is essential for future battery designs. LiPS are known to dissociate or react with each other to form intermediate species in the electrolyte [1,2]; therefore, operando characterization is necessary to understand the conditions and locations in which LiPS form before additional reactions occur. Operando X-ray absorption spectroscopy has been performed on $\mathrm{Li}-\mathrm{S}$ in both conventional and cross-sectional geometries [3-5], but literature on operando mapping is limited [6,7]. In addition, the solid electrolyte interphase (SEI), a surface film that forms on electrodes, is largely mysterious, especially in newer Li-S chemistries such as those with a polyethylene oxide binder.

In this study, ex situ and operando spectromicroscopy at the sulfur K-edge $(2472 \mathrm{eV})$ are used to study the SEI on Li metal and LiPS during cycling. All batteries consisted of Li metal foil with a sulfur/carbon composite thin film electrode (average sulfur loading $=6 \mathrm{mg} \mathrm{cm}^{-2}$ ) on carbon-coated aluminum foil. For ex situ characterization, the conventional electrolyte chemistry of $1 \mathrm{M}$ bis(trifluoromethane)sulfonimide Li salt (LiTFSI) with $0.3 \mathrm{M} \mathrm{LiNO}_{3}$ in a 1:1 mixture by volume of 1,3-dioxolane and 1,2-dimethoxyethane (DOL/DME) was used. For operando measurements, an electrolyte solution of $1 \mathrm{M} \mathrm{LiClO}_{4}$, another common battery salt, with $0.2 \mathrm{M} \mathrm{LiNO}_{3}$ in DOL/DME was used to eliminate the signal from the sulfurcontaining LiTFSI salt. Thus, any detected sulfur signal originated from the sulfur species in the electrode.

Ex situ characterization was performed on Li foil removed from a cycled coin cell to determine the composition of the surface films, shown in Figure 1. The energies in (a), (b), and (c) correspond to LiPS, $\mathrm{Li}_{2} \mathrm{~S}$, and LiTFSI, respectively. These maps demonstrate that even at low current $\left(0.04 \mathrm{~mA} \mathrm{~cm}^{-2}\right)$, active material is lost to deposition of $\mathrm{Li}_{2} \mathrm{~S}$ on the electrode surface.

Because the diffusion behavior of the LiPS across the electrolyte is of great interest, a cross-sectional cell (Figure 2) was designed to track LiPS in the separator/electrolyte and cathode region. Figure 3 shows the discharge/charge curve of a cross-sectional battery along with LiPS maps taken at $2470.35 \mathrm{eV}$ [7]. Significant microstructural changes are seen in the electrode, even during the first discharge. LiPS begin to form before cycling (a), and a significant fraction of LiPS remain in the electrode after charging, indicated by the yellow and red regions in (i). The direct observation that this material no longer 
participates in the electrochemical reaction supports the trend of decreasing capacity. At the step size used $(15 \mu \mathrm{m})$, it is difficult to resolve the LiPS at the Li/separator interface; however, by mapping a smaller area with a finer step $(5 \mu \mathrm{m}$, as in Figure 1), chemistry at the interfaces can be observed. This mapping optimization will be pursued in future work along with improvement of cell electrochemistry and capacity via pressure plates to ensure electrode contact [9].

\section{References:}

[1] H. Yamin, J. Electrochem. Soc. 135 (1988) 1045.

[2] S. Zhang, et al, Adv. Energy Mater. 5 (2015) 1500117.

[3] Y. Gorlin, et al, J. Electrochem. Soc. 163 (2016) A930.

[4] M. Cuisinier, et al, J. Phys. Chem. Lett. 4 (2013) 3227.

[5] T.A. Pascal, et al, J. Phys. Chem. Lett. 5 (2014) 1547.

[6] E.C. Miller, et al, J. Electrochem. Soc. 165 (2018) A6043.

[7] A.T.S. Freiberg, et al, J. Phys. Chem. C. 122 (2018) 5303.

[9] The authors would like to thank S. Webb, C. Roach, D. van Campen, and J.N. Weker at SSRL. This research was supported by the Sony Corporation, Murata Manufacturing, the Joint Center for Energy Storage Research, Battery500, and the DOE Summer Undergraduate Laboratory Internship Program.
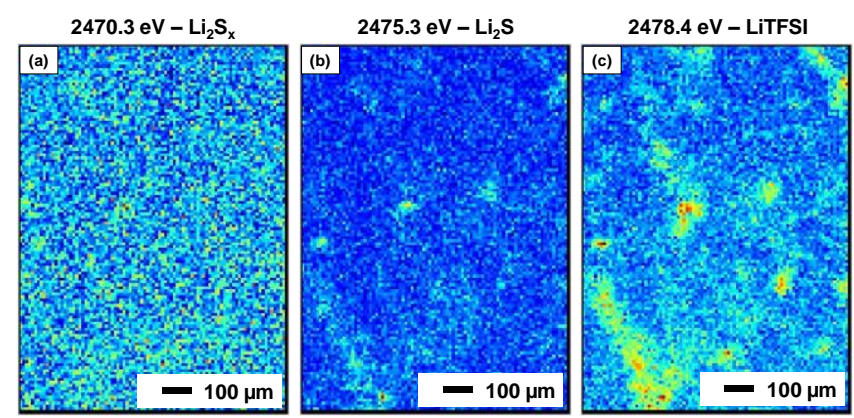

Figure. 1. Multi-energy maps of a Li surface from a cycled coin cell showing the $\mathrm{LiPS}, \mathrm{Li}_{2} \mathrm{~S}$, and LiTFSI distribution.
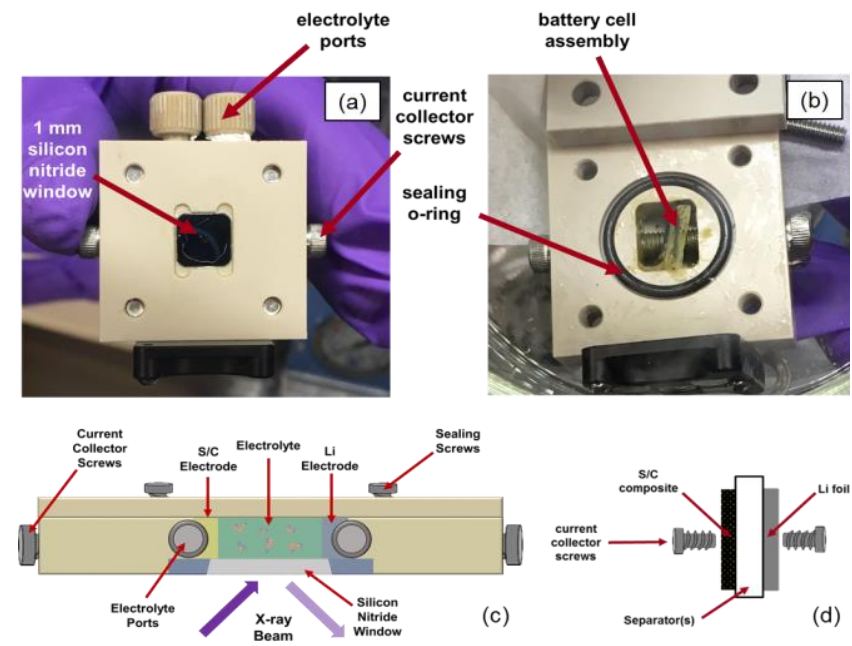

Figure. 2. Photos (a), (b) and schematics (c), (d) of the cross-sectional cell used for operando characterization. Modified from [7]

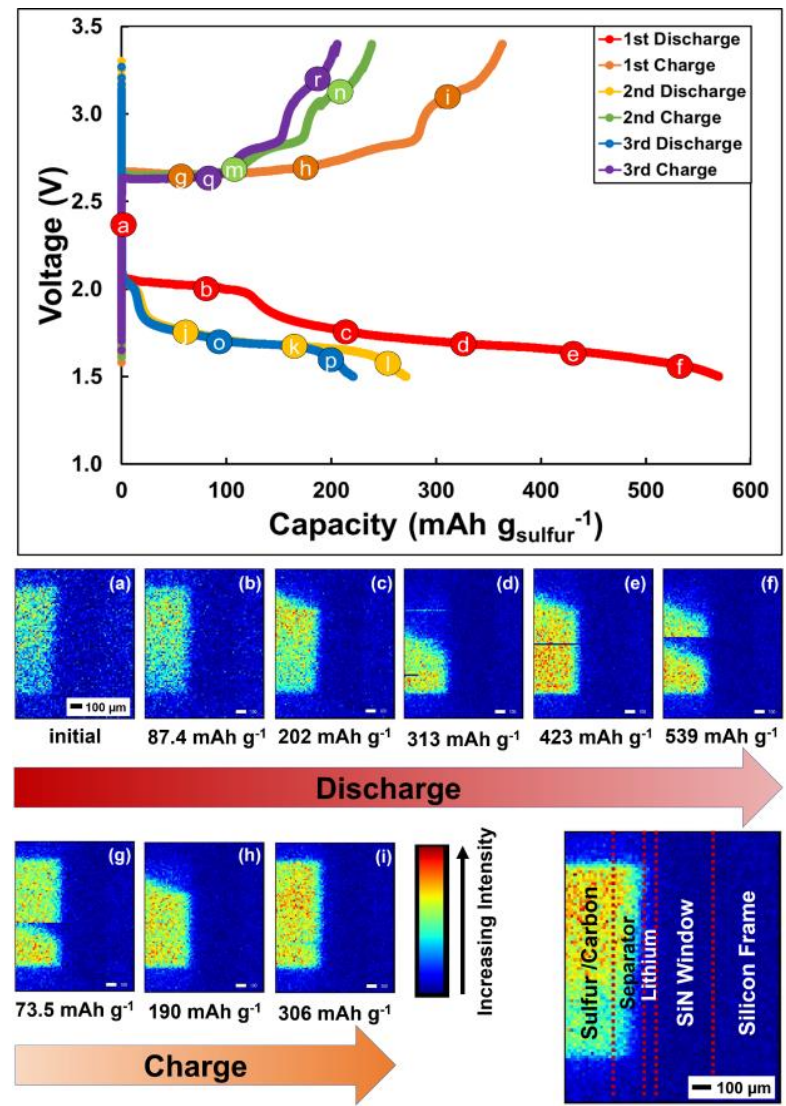

Figure. 3. Electrochemistry of the crosssectional cell with the LiPS maps at 2470.35 $\mathrm{eV}$ for the first discharge/charge cycle [7] 